\title{
Sensitivitätsanalyse zu Infiltrationsprozessen in Böden
}

\author{
Giuseppe Brunetti · Marleen Schübl · Katharina Santner · Christine Stumpp
}

Angenommen: 20. Dezember 2021 / Online publiziert: 25. Januar 2022

(c) Der/die Autor(en) 2022

Zusammenfassung Infiltrationsprozesse spielen eine wichtige Rolle bei der Entstehung von pluvialem Hochwasser. Ob Oberflächenabfluss generiert wird, hängt unter anderem von bodenhydraulischen Eigenschaften ab. Ein zukünftiger verbesserter Schutz vor pluvialem Hochwasser basiert somit auf einem verbesserten Prozessverständnis zu Infiltrationsprozessen in Böden, um Bedingungen und Parameter zu identifizieren, die zu pluvialem Hochwasser führen und die entscheidend für die Vorhersage von Ereignissen sind. Deshalb wurde das Modell KINEROS für eine globale und lokale Sensitivitätsanalyse verwendet, um Parameter zu identifizieren, die den Oberflächenabfluss am stärksten beeinflussen und um den Oberflächenabfluss in Abhängigkeit vom anfänglichen Bodensättigungsgrad für verschiedene Niederschlagsmengen an 14 Standorten in Österreich zu berechnen. Die globale Sensitivitätsanalyse hat gezeigt, dass vor allem die Faktoren gesättigte hydraulische Leitfähigkeit und Oberflächenrauheit sowohl das Gesamtabflussvolumen als auch dessen Spitze maßgeblich beeinflussen. Die Verteilung und Intensität des Niederschlags hat ebenfalls einen großen Einfluss auf den Abflussprozess. Demgegenüber haben die Hangneigung und die anfängliche Bodensättigung einen moderaten Einfluss. Auch wenn der Sättigungsgrad weniger entscheidend war, zeigt die lokale Sensitivitätsanalyse eine nichtlineare Beziehung zwischen dem anfänglichen Sättigungsgrad des Bodens und der Gesamtabflussmenge, besonders bei den mittel- und hochdurchlässigen Böden. Es konnte klar gezeigt werden, dass hydrologische

\section{Dr. G. Brunetti .}

M. Schübl, MSc. · K. Santner .

Univ.-Prof. Dr. C. Stumpp ( $\square)$ Department für

Wasser-Atmosphäre-Umwelt, Institut für Bodenphysik und landeskulturelle Wasserwirtschaft, Universität für Bodenkultur Wien, Muthgasse 18, 1190 Wien, Österreich christine.stumpp@boku.ac.at
Modelle in Verbindung mit detaillierter, statistischer Analyse ein wichtiges Instrument zur Analyse und Bewertung der hydrologischen Prozesse und deren Unsicherheiten auf der Plotskala sind.

Schlüsselwörter Bodenhydraulische Parameter · Infiltration · Pluviales Hochwasser - Bodenhydrologische Modellierung

\section{Sensitivity analysis of soil infiltration processes}

Abstract Infiltration processes play a key role in the generation of pluvial floods. Whether surface flow is generated depends, among other things, on soil hydraulic properties. Future improved protection against pluvial floods is thus based on an improved process understanding of infiltration processes in soils in order to identify conditions that lead to pluvial floods and parameter that are crucial for the prediction of events. Therefore, the KINEROS model was used for a global and local sensitivity analysis to identify parameters that most influence overland flow and to calculate overland flow as a function of initial soil saturation level for different rainfall amounts at 14 locations in Austria. The global sensitivity analysis showed that especially the factors saturated hydraulic conductivity and surface roughness significantly influence both the total runoff volume and its peak. The distribution and intensity of precipitation also have a major influence on the runoff process. In contrast, slope and initial soil saturation have a moderate impact on runoff peak and volume, respectively. Even though the degree of saturation was less crucial, the local sensitivity analysis shows a nonlinear relationship between the initial degree of soil saturation and the total runoff volume especially for the soils with medium and high saturated hydraulic conductivity. It was clearly demonstrated that hydrologic models combined with detailed statistical analysis are an important tool for analyzing and evaluating hydrologic processes and their uncertainties at the plot scale.

Keywords Soil hydraulic properties . Infiltration · Pluvial floods · Soil hydrological modelling

\section{Einleitung und Zielsetzung}

Starkniederschlagsereignisse mit pluvialem Hochwasser als Folge haben in den letzten Jahren zu hohen Sachschäden geführt und sogar Menschenleben gefordert. In Österreich sind Schäden ausgelöst durch pluviale Hochwasserereignisse und damit verbundene Kosten im ähnlichen Bereich wie die fluvialer Hochwasserereignisse (Glade et al. 2020). Ein zukünftiger verbesserter Schutz vor pluvialem Hochwasser basiert auch auf einem verbesserten Prozessverständnis zu Infiltrationsprozessen in Böden, um Bedingungen und Parameter zu identifizieren, die zu pluvialem Hochwasser führen und die entscheidend für die Vorhersage von Ereignissen sind.

Neben Intensität und Dauer der Niederschläge sowie topografischen Eigenschaften, sind Landnutzung sowie bodenhydraulische Eigenschaften sehr entscheidend dafür, wie viel Niederschlag in den Boden infiltriert oder oberflächlich abfließt. Diese Prozesse sind räumlich und zeitlich stark variabel (Meißl et al. 2020, 2021). Um diese Prozesse $\mathrm{zu}$ beschreiben und $\mathrm{zu}$ quantifizieren werden unterschiedliche Modelle herangezogen, die sich sehr stark in ihrer Komplexität und in den beschreibenden Prozessen unterscheiden und auf unterschiedlichen Skalen auch in Österreich zum Einsatz kommen (Achleitner et al. 2020). Gerade auf größerer Skala werden bodenhydrologische Prozesse bei der Simulation von Oberflächenabflüssen - wenn überhaupt - vereinfacht dargestellt, weil eben einerseits Rechenkapazitäten und die numerische Koppelung von unterschiedlichen Modellen eine Herausforderung darstellt und andererseits eben auch flächig sehr unterschiedliche Information $\mathrm{zu}$ bodenhydrauli- 
schen Parametern vorliegt (Zeitfogel et al. 2022). Selbst auf der Plotskala sind Koppelung von physikalisch basierten Modellen und deren numerische Lösung eine Herausforderung, da die Prozesse Oberflächenabfluss und Bodenwasserfluss mit unterschiedlichen Zeitskalen verbunden sind. Aber gerade die Verwendung physikalisch basierter Modelle kann helfen, Infiltrationsprozesse besser $\mathrm{zu}$ verstehen und durch Sensitivitätsanalysen die maßgeblichen Parameter zur Generierung von Oberflächenabfluss zu identifizieren. Deshalb wurde das Modell KINEROS (Goodrich et al. 2012) für eine globale und lokale Sensitivitätsanalyse herangezogen. Ziel war es, die Parameter zu identifizieren, die den Oberflächenabfluss am stärksten beeinflussen und $\mathrm{zu}$ untersuchen, ob das Modell Wechselwirkungen zwischen den Parametern enthält. Zusätzlich wurde eine lokale Sensitivitätsanalyse an 14 Standorten in Österreich mit typischen Böden durchgeführt, um den Oberflächenabfluss in Abhängigkeit vom anfänglichen Bodensättigungsgrad für verschiedene Niederschlagsmengen zu berechnen.

\section{Methoden}

Die numerische Modellierung von Oberflächenabfluss in Kombination mit Infiltrationsprozessen in porösen Medien ist eine anspruchsvolle numerische Aufgabe, da sie die Beschreibung von Prozessen beinhaltet, die auf verschiedenen Zeitskalen ablaufen. Hydrodynamisch wird der Oberflächenabfluss durch die Flachwassergleichung beschrieben, während die Richards-Gleichung den variabel gesättigten Wasserfluss in Böden parametrisiert. Die numerische Auflösung der vollständig gekoppelten partiellen Differentialgleichungen stellt zahlreiche numerische Herausforderungen dar, die unweigerlich $\mathrm{zu}$ einem hohen Rechenaufwand und einer schlechten Skalierbarkeit von Modellen führt. Dies erschwert die Verwendung für hydrologische Analysen auf Einzugsgebietsebene, die häufig mit Modellen durchgeführt werden, die die Beschreibung des Infiltrationsprozesses vorzugsweise durch konzeptionelle oder analytische Formulierungen vereinfachen. Die resultierenden Modelle haben eine geringere Anzahl von Parametern und sind rechnerisch effizient und skalierbar. Deswegen wurde hier die numerische Analyse des Oberflächenabflusses mit dem physikalisch basierten und ereignisorientierten Modell KINEROS (Goodrich et al. 2012) durchgeführt. Dieses Modell wurde für eine generelle Sensitivitätsanalyse herangezogen, um festzustellen, welche Faktoren und wie der Oberflächenabfluss beeinflusst wird. Zusätzlich und basierend auf diesen Ergebnissen wurde an 14 Standorten in Österreich, an denen Bodenwassermonitoring durch das Bundesministerium für Landwirtschaft, Regionen und Tourismus (BMLRT) betrieben wird (Gabriele Fuchs, persönlicher Kontakt; Bernsteiner et al. 2020), eine lokale, vergleichende Sensitivitätsanalyse durchgeführt, um den Oberflächenabfluss in Abhängigkeit vom anfänglichen Bodensättigungsgrad für verschiedene Niederschlagsmengen $\mathrm{zu}$ berechnen. Zur Parametrisierung der bodenhydraulischen Eigenschaften wurde eine inverse Anpassung mit dem numerischen, bodenhydrologischen Modell HYDRUS1D durchgeführt (Šimůnek et al. 2016) und entsprechend den Parameteranforderungen für KINEROS neu berechnet.

\subsection{KINEROS}

KINEROS ist ein hydrologisches Modell für Wassereinzugsgebiete, das die eindimensionale kinematische Wellengleichung verwendet, um den Abfluss über rechteckige Flächen und durch trapezförmige offene Kanäle, kreisförmige Kanäle und kleine Rückhaltebecken zu simulieren. Der Infiltrationsalgorithmus ist dynamisch und interagiert sowohl mit dem Niederschlag als auch mit dem durchfließenden Oberflächenwasser. Er basiert auf einer physikalisch begründeten Annäherung an den Infiltrationsprozess, in dem ein zweischichtiges Bodenprofil angenommen werden kann (Smith 1990; Smith et al. 1993; Corradini et al. 1994). Diese Formulierung erfordert die Definition von vier Parametern für jede Bodenschicht: die gesättigte hydraulische Leitfähigkeit $(K s[\mathrm{~L} / \mathrm{T}])$, die mittlere Wirkung des Kapillarflusses $(G[L])$, der Index der Porengrößenverteilung $(D[-])$ und die Porosität $\left(n\left[\mathrm{~L}^{3} / \mathrm{L}^{3}\right]\right)$. Diese Parameter können für verschiedene Bodenbeschaffenheitsklassen geschätzt werden und sind tabellarisch verfügbar (Rawls et al. 1982); für die vorliegende Arbeit wurden typische Bandbreiten der Parameter für die Sensitivitätsanalyse benutzt (siehe Abschn. 2.3). Darüber hinaus müssen der anfängliche Sättigungsgrad des Bodens $\left(\Theta\left[\mathrm{L}^{3} / \mathrm{L}^{3}\right]\right)$ und die Mächtigkeit der oberen Bodenschicht $(d[\mathrm{~L}])$ definiert werden.

\subsection{Randbedingungen}

Zur Durchführung der ereignisbasierten hydrologischen Analysen werden neben den hydraulischen Eigenschaften des Bodens zusätzlich die morphologischen Eigenschaften des Einzugsgebiets (z. B. Gefälle, Rauheit) und die Niederschlagszeitreihen benötigt. Letztere können gemessen oder mithilfe mathematischer Formeln, die durch wenige Parameter gekennzeichnet sind, synthetisch erzeugt werden. In der vorliegenden Studie werden synthetische Niederschlagszeitreihen unter Verwendung der von Carbone et al. (2015) vorgeschlagenen doppelexponentiellen Formulierung erzeugt:

$$
F(t)=v o l \cdot e^{-e^{-\alpha(t-\beta)}}
$$

wobei $F(t)$ die kumulative Niederschlagsverteilungsfunktion $[\mathrm{L}]$ ist, $v o l$ die in der Zeit $t[\mathrm{~T}]$ verteilte Niederschlagsmenge [L] ist und $\alpha$ und $\beta$ zwei empirische Parameter [-] sind, die die Kurtosis und Schiefe des Niederschlags beschreiben (Abb. 1). Die Verwendung eines synthetischen Niederschlags ist für die folgende Sensitivitätsanalyse von praktischer Bedeutung, da sie es ermöglicht, die Eigenschaften des Niederschlags in nur drei Parametern zusammenzufassen und ihren Einfluss auf das hydrologische Verhalten des Systems zu untersuchen.

\subsection{Globale Sensitivitätsanalyse}

Das Hauptziel dieser Aufgabe ist die Durchführung einer Sensitivitätsanalyse (SA), um festzustellen, welche Faktoren wie den Oberflächenabfluss beeinflussen. $\mathrm{Zu}$ diesem Zweck wurde KINEROS verwendet, um die hydrologischen Prozesse in einem einzelnen Einzugsgebiet $(100 \times 100 \mathrm{~m})$ bei Variation mehrerer Faktoren zu simulieren. Die Analyse umfasste insbesondere Faktoren, die die Geometrie des Einzugsgebiets (d.h. Neigung, ManningKoeffizient), das Niederschlagsregime (d.h. vol, $\alpha$ und $\beta$ ) und die Bodeneigenschaften (d.h. $\Theta$ und $K s, G, D, n$ für zwei Bodenschichten (1 und 2)) beschreiben, was zu insgesamt 14 Parametern führte und typische Bandbreiten an Parameterwerten beinhaltete: vol: $5-60 \mathrm{~mm}$, $\alpha: 0,05-0,4, \beta: 5-120$, Neigung: $1-50 \%$, Manning-Koeffizient: $0,01-0,7 \mathrm{sm}^{-1 / 3}, \Theta$ : 

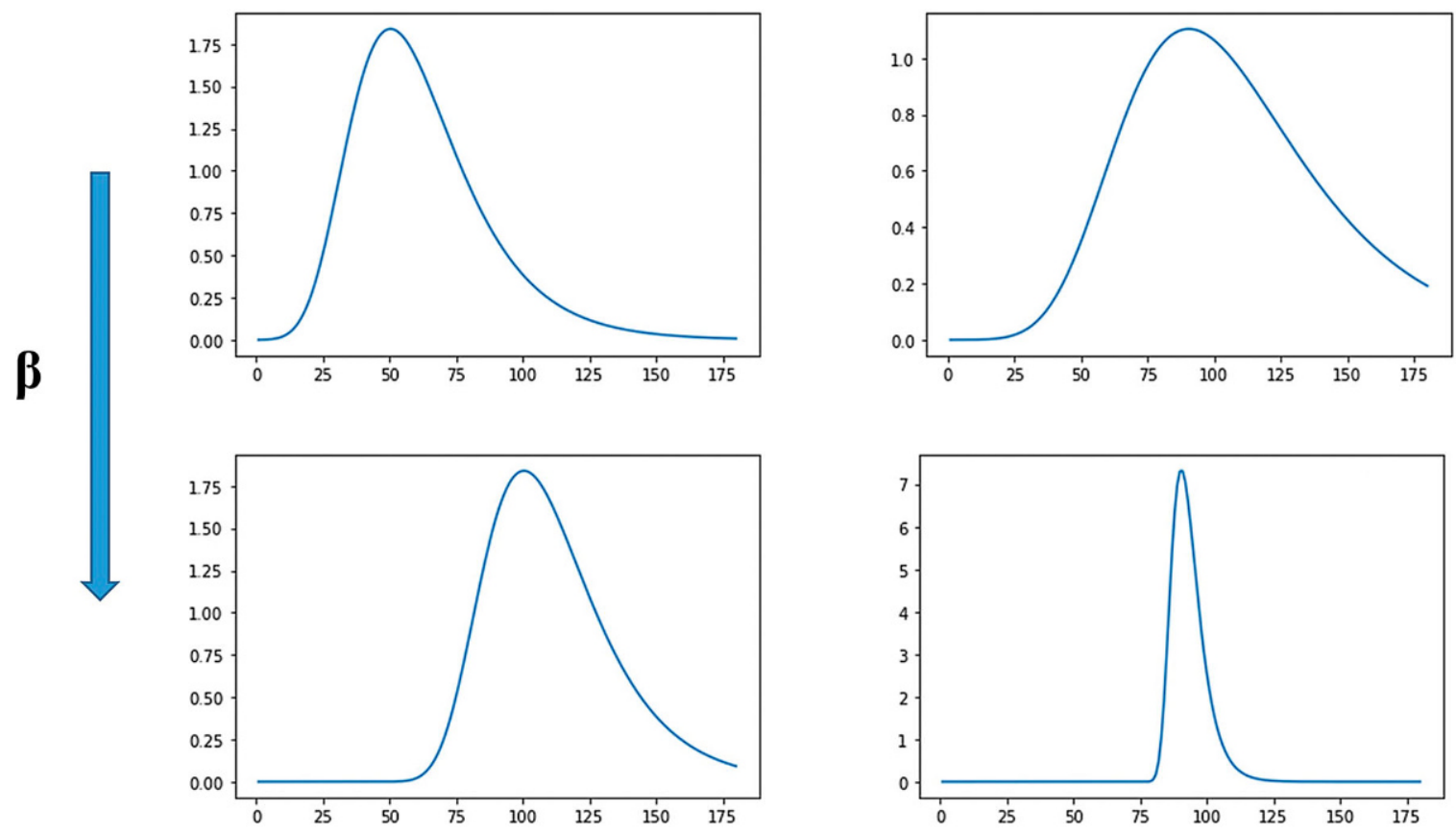

Abb. 1 Auswirkungen der Parameter $\alpha$ (rechts) und $\beta$ (links) auf den synthetischen Niederschlag

5-99\%, $K s_{1,2}: 1-300 \mathrm{~cm} / \mathrm{d}, G_{1,2}: 5-40 \mathrm{~cm}$, $D_{1,2}: 0,1-0,7$ und $n_{1,2}: 0,3-0,6$.

Bei den meisten in der Literatur durchgeführten SAs handelt es sich um sogenannte "One-at-a-time“ (OAT)Sensitivitätsanalysen, bei denen der Wert eines Parameters nach dem anderen geändert wird, während die anderen konstant bleiben (Cheviron und Coquet 2009; Houska et al. 2014; Rezaei et al. 2016). Wenn das Modell jedoch Wechselwirkungen zwischen den Parametern enthält, sind die Ergebnisse der OATAnalyse ungenau, da die Wechselwirkungen der Parameter nur durch die gleichzeitige Änderung mehrerer Parameter ermittelt werden können. Aus diesem Grund wird, wenn die Eigenschaft eines Modells a priori unbekannt ist, immer eine globale Sensitivitätsanalyse (GSA) bevorzugt (Saltelli und Annoni 2010).

Einer der am meisten verbreiteten Algorithmen für die GSA ist die varianzbasierte Sobol-Methode (Sobol 2001). Varianzbasierte Methoden zielen darauf ab, den Betrag der Varianz zu quantifizieren, den jeder Parameter zur unbedingten Varianz der Modellausgabe beiträgt. Bei der Sobol-Methode werden diese Beiträge durch die Sobol-Sensitivitätsindizes (SIs) dargestellt. Diese Indizes geben quantitative Informationen über die Varianz, die mit einem einzelnen Parameter oder mit den Wechselwirkungen zwischen meh- reren Parametern verbunden ist. Eine ausführlichere Erläuterung der SobolMethode findet sich in Sobol (2001).

Die Sobol'schen Sensitivitätsindizes werden wie folgt ausgedrückt:

$$
\begin{aligned}
& \text { ErsterOrdnung } S_{i}=\frac{V_{i}}{V} \\
& \text { ZweiterOrdnung } S_{i i}=\frac{V_{i j}}{V} \\
& \text { Gesamt }_{T}=S_{i}+\sum_{j \neq i} S_{i j}+\ldots
\end{aligned}
$$

wobei $V i$ die mit dem $i$-ten Parameter verbundene Varianz und $V$ die Gesamtvarianz ist. Der Index erster Ordnung, $S_{i}$, wird in der Literatur als „Haupteffekt" bezeichnet. Dieser Index kann als der Anteil der Varianz der Modellausgabe beschrieben werden, der verschwindet, wenn der Parameter $X_{i}$ festgelegt wird. Wenn das Modell additiv ist, d.h., wenn es keine Wechselwirkungen zwischen den Inputfaktoren enthält, ist der Index erster Ordnung ausreichend, um die Varianz des Modells zu zerlegen. Für additive Modelle gilt die folgende Beziehung:

$$
\sum_{i} S_{i}=1
$$

Selbst wenn das Modell Wechselwirkungen zwischen den Parametern enthält, bleibt der Index erster Ordnung das Maß, das für die Priorisierung der Faktoren verwendet werden sollte (Saltelli et al. 2004). Andererseits gibt der Index der Gesamtwirkung $\left(S_{T}\right)$ Aufschluss über einen nicht-additiven Teil des Modells. Ein signifikanter Unterschied zwischen $S_{T}$ und $S_{i}$ weist auf eine wichtige Rolle einer Interaktion für den betrachteten Parameter hin. Im Wesentlichen gibt der Gesamteffektin$\operatorname{dex} S_{T i}$ den Anteil der Gesamtvarianz an, der übrig bliebe, wenn alle Faktoren außer $X_{i}$ fixiert wären. $S_{T i}=0$ ist eine notwendige und hinreichende Bedingung dafür, dass $X_{i}$ nicht einflussreich ist. Daher kann $X_{i}$ auf einen beliebigen Wert innerhalb seines Unsicherheitsbereichs festgelegt werden, ohne die unbedingte Varianz des Outputs zu beeinflussen. Der Gesamteffekt ist das Maß, das für die Festlegung der Faktoren zu verwenden ist.

In Anbetracht der Tatsache, dass hydrologische Modelle im Allgemeinen stark nichtlinear sind, ist es fast unmöglich, die Varianzen mit analytischen Integralen zu berechnen. Daher werden häufig Monte-Carlo-Integrale angewandt, die auf einer Abtastung des Parameterraums in $q$ Stichproben beruhen. Es liegt auf der Hand, dass die Genauigkeit bei der Schätzung von Integralen mit zunehmender Anzahl von Stichproben steigt, was auch die Rechenkosten der SA erhöht. Eine genaue Beschreibung der Berechnung der Sobol-Indizes findet sich in Saltelli et al. (2010). 
Grundsätzlich erfordert die Berechnung der Sobol-Indizes $q \cdot(2 p+1)$ Modellevaluierungen, wobei $p$ die Anzahl der Eingabefaktoren ist. Saltelli (2002) führte jedoch eine Methode ein, die nur $q \cdot(p+2)$ Modellevaluierungen erfordert. Zur Abtastung des Parameterraums haben wir die Quasi-Zufallsstichprobentechnik von Sobol (Sobol 2001) verwendet. Einer der wichtigsten Aspekte der GSA ist die Wahl der Anzahl der Stichproben, $q$. Eine Erhöhung der Anzahl der Stichproben erhöht die Genauigkeit der Sobol-Indizes. Ein hohes q bedeutet jedoch auch eine höhere Anzahl von Modellevaluierungen. Die Anzahl der Stichproben hängt von der Struktur des Modells und von der Art der durchgeführten Simulationen ab. Im vorliegenden Projekt wurde $q$ auf 1000 festgelegt, was als angemessene Zahl für die Dimensionalität des untersuchten Problems angesehen wird.

Um die Genauigkeit der Schätzungen der Sensitivitätsindizes zu bewerten, wurden die Bootstrap-Konfidenzintervalle (BCI) (Efron und Tibshirani 1986) geschätzt. Der Grundgedanke des Bootstrapping besteht darin, dass die Stichprobe in Ermangelung anderer Informationen über die Verteilung alle verfügbaren Informationen über die zugrundeliegende Verteilung enthält. In unserem speziellen Fall waren wir daran interessiert, die Unsicherheit der geschätzten Sensitivitätsindizes zu berechnen. Da ihre Verteilung jedoch unbekannt ist, ist es nicht möglich, die Konfidenzintervalle analytisch zu berechnen. Das Grundprinzip der Bootstrap-Methode besteht darin, die unbekannte Verteilung durch ihre empirische Verteilung $\mathrm{zu}$ ersetzen und die Sensitivitätsindizes mithilfe eines Monte-Carlo-Simulationsansatzes zu berechnen, bei dem Stichproben durch Wiederholung der für die Sensitivitäts-

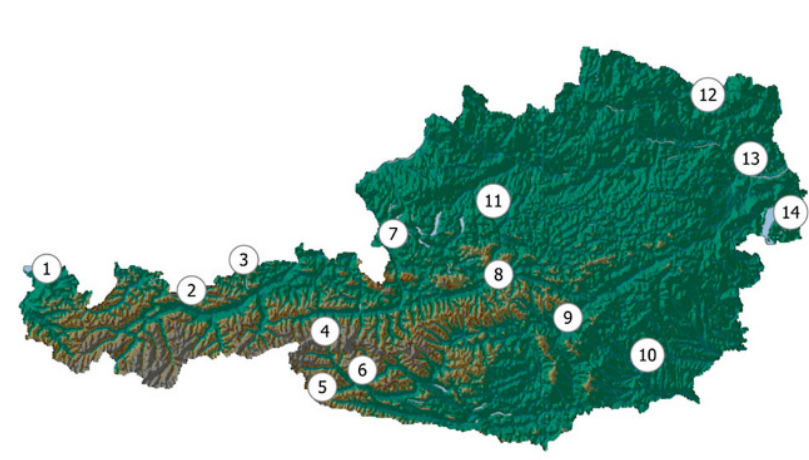

1. Lauterach

2. Leutasch

3. Achenkirch

4. Gschlössboden

5. Sillianberger Alm

6. Zettersfeld

7. Elsbethen

8. Gumpenstein

9. Aichfeld-Murboden

10. Kalsdorf

11. Pettenbach

12. Schalladorf

13. Lobau

14. Frauenkirchen
Für jeden der 14 Standorte wurde mit der Software HYDRUS-1D ein eindimensionales numerisches Modell zur Beschreibung der bodenhydrologischen Wasserflüsse in der ungesättigten Zone erstellt. Aus den langjährigen Datenreihen (Bodenwassergehalt und Matrixpotenzial) wurde ein Zeitraum (unter schneefreien Bedingungen) mit möglichst vollständigen und plausiblen Daten ausgewählt und für die inverse Bestimmung der bodenhydraulischen Parameter des Modells durchgeführt. Die Parametrisierung erfolgt über den van-Genuchten bzw. den Mualem-vanGenuchten-Ansatz für die Wasserretentions- und die Leitfähigkeitsfunktion (van Genuchten 1980). Zuerst wurden die Parameter mit einem Particle Swarm-Algorithmus über eine kombinierte Zielfunktion für Wassergehalt und Matrixpotenzial optimiert. Die optimierten Werte dienen als Ausgangspunkte für die anschließende Markov Chain Monte Carlo (MCMC)-Kalibrierung, bei der die sich aus den Datenund Modellstrukturunsicherheiten ergebenden Parameterunsicherheiten bestimmt wurden. Dies basiert auf dem Bayes'schen Ansatz, bei dem die A-prio$r i$-Wahrscheinlichkeiten der Parameterwerte (physikalisch plausibler Bereich bzw. basierend auf Angaben zur Bodentextur) mit den Wahrscheinlichkeiten aus der Modellevaluation (Vergleich mit Messdaten) kombiniert wurden, um so die A-posteriori-Wahrscheinlichkeitsverteilung der Parameterwerte zu berechnen. Diese Parameterwerte wurden entsprechend den Parameteranforderungen für KINEROS umgewandelt, gemäß den Ergebnissen der globalen Sensitivitätsanalyse vereinfacht und es wurde eine lokale Sensitivitätsanalyse an den 14 Standorten durchgeführt. Folgende Parameter wurden gewählt: $\alpha=0,1, \beta=60$, Neigung $=10 \%$, ManningKoeffizient $=0,15 \mathrm{~s} \mathrm{\quad m^{-1/3 }}, \quad G=50 \mathrm{~mm}$, $D=0,1, n=0,4$. Außerdem wurden der Median der hydraulischen Leitfähigkeit aus HYDRUS-1D und die Mächtigkeit der oberen Bodenschicht gemäß Tab. 1 übernommen. Das Niederschlagsvolumen vol und der Sättigungsgrad $\Theta$ wurden für ein 180 minutiges Niederschlagsereignis variiert.

\section{Ergebnisse und Diskussion}

\subsection{Globale Sensitivitätsanalyse}

Die geschätzten Indizes der Wirkung erster Ordnung $\left(S_{I}\right)$ und der Gesamt-

Abb. 2 14 Standorte des Bodenwassermonitorings in Österreich 
Tab. 1 Invers kalibrierte gesättigte hydraulische Leitfähigkeiten und Unsicherheitsbereiche $(2,5 \%, 97,5 \%)$ der 14 Monitoring-Standorte

\begin{tabular}{|c|c|c|c|c|}
\hline \multirow[t]{2}{*}{ Standort } & \multirow[t]{2}{*}{ Bodenhorizont } & \multicolumn{3}{|c|}{$\mathrm{Ks}[\mathrm{cm} / \mathrm{d}]$} \\
\hline & & Median & $2,5 \%$ & $97,5 \%$ \\
\hline 1. Lauterach & $0-80 \mathrm{~cm}$ & 50,3 & 34,8 & 235,3 \\
\hline \multirow[t]{2}{*}{ 2. Leutasch } & $0-35 \mathrm{~cm}$ & 23,5 & 13,3 & 53,8 \\
\hline & $35-140 \mathrm{~cm}$ & 310,0 & 148,8 & 865,9 \\
\hline \multirow[t]{2}{*}{ 3. Achenkirch } & $0-15 \mathrm{~cm}$ & 21,1 & 12,5 & 32,7 \\
\hline & $15-70 \mathrm{~cm}$ & 360,2 & 248,9 & 486,6 \\
\hline 4. Gschlössboden & $0-35 \mathrm{~cm}$ & 3012,5 & 1053,5 & 4891,3 \\
\hline 5. Sillianberger Alm & $0-55 \mathrm{~cm}$ & 424,8 & 270,3 & 659,2 \\
\hline 6. Thurn-Zettersfeld & $0-60 \mathrm{~cm}$ & 61,1 & 24,4 & 166,8 \\
\hline \multirow[t]{2}{*}{ 7. Elsbethen } & $0-25 \mathrm{~cm}$ & 20,2 & 0,5 & 1107,5 \\
\hline & $25-70 \mathrm{~cm}$ & 0,3 & 0,1 & 20,2 \\
\hline 8. Gumpenstein (Lys) & $0-150 \mathrm{~cm}$ & 13,9 & 4,9 & 44,1 \\
\hline \multirow[t]{2}{*}{ 9. Aichfeld-Murboden } & $0-35 \mathrm{~cm}$ & 3,1 & 0,9 & 15,3 \\
\hline & $35-100 \mathrm{~cm}$ & 43,9 & 20,1 & 108,5 \\
\hline \multirow[t]{2}{*}{ 10. Kalsdorf } & $0-30 \mathrm{~cm}$ & 2,2 & 1,5 & 40,0 \\
\hline & $30-150 \mathrm{~cm}$ & 1454,0 & 902,0 & 2327,0 \\
\hline \multirow[t]{2}{*}{ 11. Pettenbach } & $0-25 \mathrm{~cm}$ & 38,0 & 11,3 & 130,2 \\
\hline & $25-150 \mathrm{~cm}$ & 17,7 & 7,1 & 82,2 \\
\hline \multirow[t]{2}{*}{ 12. Schalladorf } & $0-40 \mathrm{~cm}$ & 2,2 & 1,5 & 3,9 \\
\hline & $40-150 \mathrm{~cm}$ & 2,8 & 1,1 & 6,3 \\
\hline 13. Lobau & $0-150 \mathrm{~cm}$ & 1,1 & 0,9 & 1,2 \\
\hline 14. Frauenkirchen & $0-145 \mathrm{~cm}$ & 11,7 & 7,6 & 19,2 \\
\hline
\end{tabular}

wirkung $\left(S_{T}\right)$ für die simulierte Abflussmenge und -spitze sind in Tab. 2 aufgeführt. Erstere zeigen, dass hauptsächlich vier Parameter (d.h. Ks1, vol, Manning und $\alpha$ ) einen direkten Einfluss auf das Oberflächenabflussvolumen und den Spitzenwert haben. Insbesondere die gesättigte hydraulische Leitfähigkeit Ksl der Bodenoberflächenschicht weist den höchsten Index erster Ordnung $S_{1}$ sowohl für die Abflussmenge als auch für den Spitzenwert auf. Die- ses Verhalten ist zu erwarten, da die hydraulische Leitfähigkeit des Bodens eine Hauptrolle im Infiltrationsprozess spielt. Die Niederschlagsmenge vol zeigt ähnliche Auswirkungen auf die Abflussmenge und den Spitzenwert, während der Formparameter $\alpha$ einen stärkeren Einfluss auf letzteren hat. $\alpha$ beschreibt die Kurtosis des Niederschlags (Gl. 1), die direkt mit der Form der Abflussganglinie zusammenhängt. Die Analyse bestätigt, dass Regenereig-

Tab. 2 Geschätzte Indizes der Wirkung erster Ordnung (S1) und der Gesamtwirkung (ST) für simulierte Abflussmenge und -spitze

\begin{tabular}{|c|c|c|c|c|}
\hline \multirow[t]{2}{*}{ Parameter } & \multicolumn{2}{|c|}{ Abflussmenge } & \multicolumn{2}{|c|}{ Abflussspitze } \\
\hline & $S_{1}$ & $S_{T}$ & $S_{1}$ & $S_{T}$ \\
\hline Vol & 0,20 & 0,50 & 0,20 & 0,50 \\
\hline$\alpha$ & 0,02 & 0,10 & 0,06 & 0,27 \\
\hline$\beta$ & 0,00 & 0,02 & 0,00 & 0,03 \\
\hline Neigung & 0,00 & 0,01 & 0,00 & 0,05 \\
\hline Manning & 0,01 & 0,02 & 0,04 & 0,12 \\
\hline$\Theta$ & 0,00 & 0,08 & 0,00 & 0,08 \\
\hline$K s_{1}$ & 0,46 & 0,73 & 0,27 & 0,53 \\
\hline$G_{1}$ & 0,00 & 0,03 & 0,00 & 0,03 \\
\hline$D_{1}$ & 0,00 & 0,01 & 0,00 & 0,03 \\
\hline$n_{1}$ & 0,00 & 0,03 & 0,00 & 0,03 \\
\hline$K_{s 2}$ & 0,00 & 0,12 & 0,00 & 0,08 \\
\hline$G_{2}$ & 0,00 & 0,02 & 0,00 & 0,02 \\
\hline$D_{2}$ & 0,00 & 0,00 & 0,00 & 0,00 \\
\hline$n_{2}$ & 0,00 & 0,01 & 0,00 & 0,01 \\
\hline
\end{tabular}

nisse, die durch hohe Spitzenwerte gekennzeichnet sind, im Allgemeinen zu hohen Spitzenwerten des Oberflächenabflusses führen. Schließlich hat der Manning-Koeffizient einen geringen, aber immer noch spürbaren Einfluss auf die Abflussmenge und den Spitzenwert. Eine hohe Oberflächenrauheit verringert die Oberflächenabflussgeschwindigkeit, wodurch sich die Zeit für die Wasseraufnahme durch den Boden verlängert. Gleichzeitig erhöht eine glattere Oberfläche die Oberflächenabflussgeschwindigkeit, was zu einer geringeren Infiltration und einer höheren Abflussspitze führt. Tab. 2 zeigt auch, dass die Summe aller Indizes erster Ordnung kleiner als 1 ist, was bedeutet, dass das Modell nicht additiv ist. Nur $67 \%$ der Varianz ist auf die Effekte erster Ordnung zurückzuführen, was darauf hindeutet, dass Wechselwirkungen zwischen den Parametern eine wesentliche Rolle spielen.

Dieses Verhalten spiegelt sich in den geschätzten Gesamteffektindizes $\left(S_{T}\right)$ wider, deren Summe sowohl für die Abflussmenge als auch für die Abflussspitze $>1$ ist, was auf eine starke Interaktion der Parameter hinweist. Der einflussreichste Parameter ist nach wie vor die gesättigte hydraulische Leitfähigkeit der Oberflächenschicht, $K s 1$, gefolgt von den Niederschlagseigenschaften $v o l$ und $\alpha$. Interessanterweise ist die Wirkung der zweiten Bodenschicht begrenzt und wird hauptsächlich durch die gesättigte hydraulische Leitfähigkeit $K s 2$ repräsentiert, während die anderen hydraulischen Parameter einen vernachlässigbaren Einfluss haben. Das Gefälle des Einzugsgebiets hat einen beträchtlichen Einfluss auf die Abflussspitze, ebenso wie der Manning-Koeffizient. Schließlich hat die anfängliche Bodensättigung $\Theta$, die mit der vorangegangenen Trockenwetterperiode zusammenhängt, den gleichen Gesamteinfluss auf die Abflussmenge und -spitze.

\subsection{Lokale Sensitivitätsanalyse}

Der Einfluss auf das Abflussvolumen wurde durch eine lokale Sensitivitätsanalyse genauer untersucht. Insbesondere wurde das Modell KINEROS verwendet, um eine Sensitivitätskarte zu berechnen, die die Auswirkung einer Veränderung von $\Theta$ auf das Gesamtabflussvolumen darstellt. Die Ergebnisse wurden erzielt, indem $\Theta$ verändert und alle anderen Parameter beibehalten 


\section{Originalarbeit}
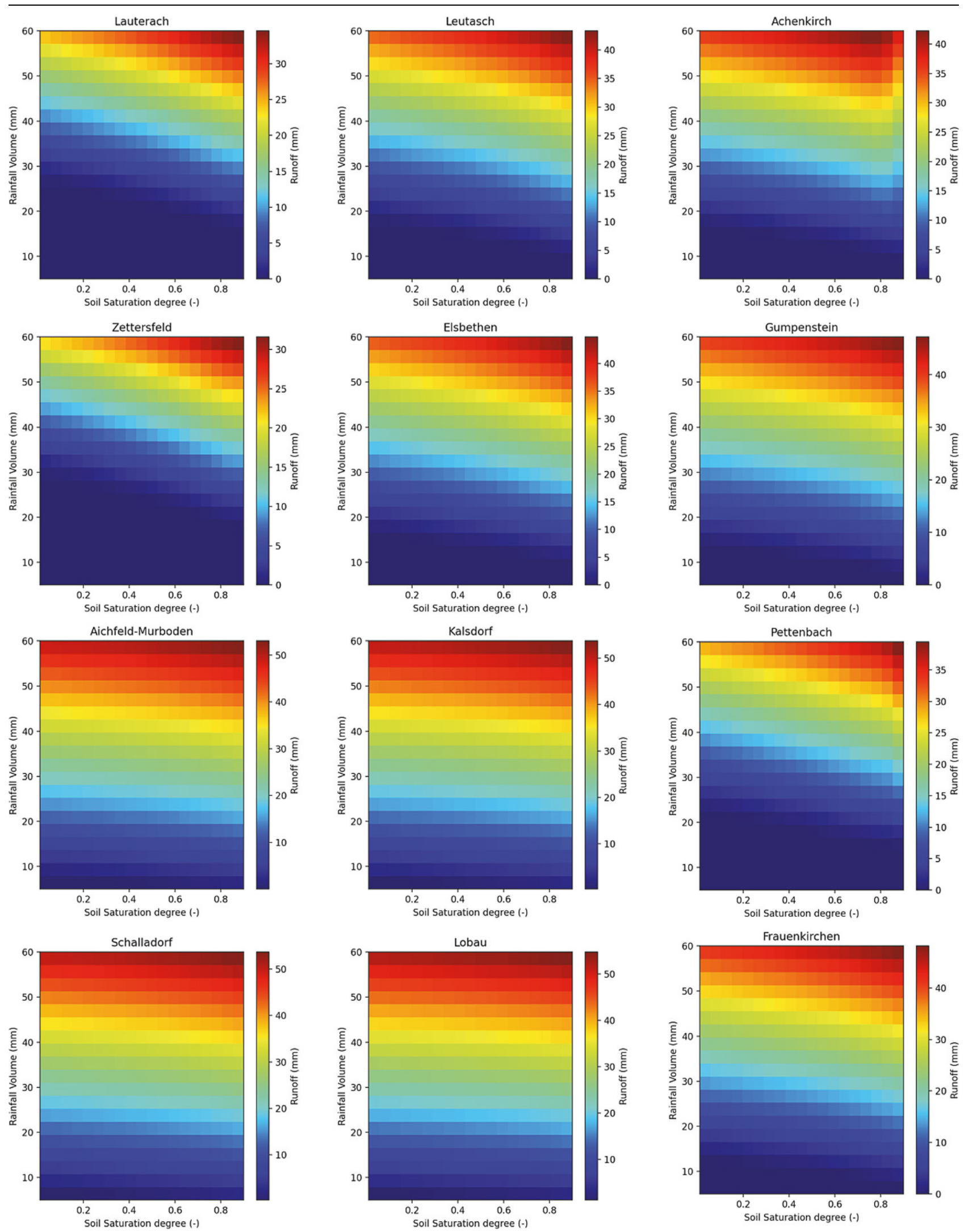

Abb. 3 Einfluss der anfänglichen Bodensättigung auf das Abflussvolumen der getesteten Standorte 
wurden. Die Analyse wurde für alle 14 Messstellen in Österreich durchgeführt (Abb. 3), wobei für die zwei Standorte Gschlössboden und Sillianberger Alm mit sehr hohen Ks-Werten (Tab. 1) kein Oberflächenabfluss in den Simulationen gefunden wurde und somit die Ergebnisse nicht dargestellt sind.

Die Ergebnisse deuten auf eine nichtlineare Beziehung zwischen dem anfänglichen Sättigungsgrad des Bodens und der Gesamtabflussmenge hin. Dies ist besonders deutlich bei den mittel- und hochdurchlässigen Böden (z.B. Leutasch und Lauterach in Abb. 3). Die Abflussmenge ist positiv mit der anfänglichen Bodensättigung korreliert, was bedeutet, dass feuchtere Böden zu einem höheren Abfluss führen. Dies ist hauptsächlich auf die Abnahme des Rückhaltevermögens des Bodens beim Übergang von trockenen zu feuchten Bedingungen zurückzuführen. Die nichtlineare Beziehung zwischen der anfänglichen Bodenfeuchte und der Abflussmenge, die für die Standorte Leutasch und Lauterach beobachtet wurde, ist hauptsächlich auf den Einfluss der zweiten Bodenschicht zurückzuführen. Dennoch zeigen die Ergebnisse der Analyse, dass eine höhere Anfangsbodenfeuchte mit einer höheren Abflussmenge korreliert; die Änderung der Menge an Oberflächenabfluss gegenüber der Bodenvorfeuchte ist allerdings weniger stark ausgeprägt (Farbverlauf entlang der x-Achse) als für verschiedene Niederschlagsmengen (Farbverlauf entlang der y-Achse) oder verschiedene Ks-Werte (Vergleich der einzelnen Standorte). Somit werden die Ergebnisse der globalen Sensitivitätsanalyse auch für die lokale Analyse bestätigt. Das heißt, dass der Sättigungsgrad kurzfristig und lokal schon eine Rolle bei der Entstehung pluvialer Hochwasserereignisse hat, aber die Variabilität der hydraulischen Leitfähigkeit oder die Niederschlagsmenge wesentlich entscheidender ist. Für Böden mit sehr niedriger hydraulischer Leitfähigkeit ist selbst bei geringem Sättigungsgrad die Infiltration der hier getesteten Niederschlagsereignisse sehr gering, sodass ein hoher Teil des Wassers theoretisch oberflächlich abfließen würde (z.B. Lobau, Schalladorf). Böden mit sehr hohen Ks-Werten zeigen selbst bei sehr hohen Niederschlagsereignissen keine Generierung von Oberflächenabfluss (z.B. Sillianberger Alm; Daten nicht in Abbildung).

\subsection{Synthese}

Unter der Berücksichtigung der Tatsache, dass Ks-Werte nur mit großen Unsicherheiten hervorgesagt werden können (Zeitfogel et al. 2022), sollten Methoden entwickelt werden, hydraulische Leitfähigkeiten besser vorhersagen zu können. Pedotransferfunktionen eignen sich, um auf regionaler Ebene Vorhersagen zu machen (Zeitfogel et al. 2022; Wallner 2021). Auf lokaler Ebene können diese auch teilweise bessere Vorhersagen liefern als Messungen im Labor (Wallner 2021), die nicht zwingend widerspiegeln, was wirklich im Feld stattfindet. Des Weiteren könnte ein effektives Monitoring von Prozessen auf der lokalen Ebene hilfreich sein, das Verständnis zur Erstehung pluvialer Hochwasserereignisse zu erhöhen. Eine Möglichkeit hierfür wäre ein kombiniertes Monitoring bodenhydrologischer Variablen, wie es u.a. an den hier gezeigten Stationen in Österreich stattfindet (Bernsteiner et al. 2020), mit Monitoring des Oberflächenabflusses unter sehr variablen Randbedingungen inklusive zusätzlicher Beregnungsexperimente zur Untersuchung von Starkregenereignissen (Ries et al. 2020; Markart et al. 2011). Zusätzlich erhöht die Weiterentwicklung von Modellen mit Integrierung des dynamischen Bodenwasserhaushalts auch die Vorhersage von Wasserflüssen und kann in einer Vielzahl von Anwendungen, wo Infiltrationsprozesse entscheidend sind, von Vorteil sein (Steinbrich et al. 2021). Langzeitversuche in Kombination mit physikalisch basierten Modellen der Hydraulik gekoppelt mit bodenhydrologischen Modellen könnten maßgeblich dazu beitragen, Infiltrationsprozesse und Oberflächenabfluss besser $\mathrm{zu}$ verstehen und eben auch Risikoabschätzungen auf lokaler Ebene durchzuführen; nicht nur in Bezug auf den Oberflächenabfluss, sondern auf die damit verbundene Erosion und allgemein für Niederschlags-AbflussBeziehungen.

\section{Schlussfolgerung}

Zum besseren Verständnis der beeinflussenden Faktoren zur Generierung von Oberflächenabfluss sind physikalisch basierte Modelle hilfreich, die Prozesse auf der Plotskala näher untersuchen. Hierfür wurden Simulationen und Sensitivitätsanalysen mit dem Modell KINEROS durchgeführt. Es zeigte sich, dass insbesondere die gesättigte Leitfähigkeit und die Oberflächenrauheit sowohl das Gesamtabflussvolumen als auch dessen Spitze maßgeblich beeinflussen. Dies legt nahe, dass neben Bodeneigenschaften auch die Landnutzung und -bewirtschaftung bei der Durchführung einer Umweltrisikobewertung berücksichtigt werden sollten. Außerdem sollten Methoden entwickelt werden, um die hydraulische Leitfähigkeit lokal und regional zu erfassen und die Unsicherheiten zu reduzieren.

Die Verteilung des Niederschlags hat einen großen Einfluss auf den Abflussprozess. Insbesondere die Intensität des Niederschlagsereignisses korreliert direkt mit der Abflusscharakteristik. Die durch den Klimawandel prognostizierte Verschiebung hin zu intensiveren Niederschlagsereignissen wird unweigerlich zu einem höheren Abflussvolumen führen.

Es konnte klar gezeigt werden, dass hydrologische Modelle in Verbindung mit detaillierter, statistischer Analyse ein wichtiges Instrument zur Analyse und Bewertung der hydrologischen Prozesse und deren Unsicherheiten auf der Plotskala sind. Ihr Einsatz sollte durch In-situ-Messungen ergänzt werden, um ihre Vorhersagefähigkeiten zu verbessern und Entscheidungsträger:innen wichtige Informationen für die Definition eines nachhaltigen Landmanagementplans zu liefern.

Danksagung Diese Arbeit ist Teil der Projekte InfCapAT - Modul „Bodenspezifische Infiltrationskapazität in Abhängigkeit der Vorfeuchte und Niederschlagsmengen" finanziert durch das BMLRT (Sektion 1 - Wasserwirtschaft, Abteilung I/6 - Hochwasserrisikomanagement) und „RechAUT - Variability of Groundwater Recharge and its Implication for Sustainable Land Use in Austria" finanziert von der Österreichischen Akademie der Wissenschaften im Rahmen des Earth System Science Programms „Wasser in Gebirgsräumen“.

Funding Open access funding provided by University of Natural Resources and Life Sciences Vienna (BOKU).

Open Access Dieser Artikel wird unter der Creative Commons Namensnennung 4.0 International Lizenz veröffentlicht, welche die Nutzung, Vervielfältigung, Bearbeitung, Verbreitung und Wiedergabe in jeglichem Medium und Format erlaubt, sofern Sie den/die ur- 
sprünglichen Autor(en) und die Quelle ordnungsgemäß nennen, einen Link zur Creative Commons Lizenz beifügen und angeben, ob Änderungen vorgenommen wurden.

Die in diesem Artikel enthaltenen Bilder und sonstiges Drittmaterial unter- liegen ebenfalls der genannten Creative Commons Lizenz, sofern sich aus der Abbildungslegende nichts anderes ergibt. Sofern das betreffende Material nicht unter der genannten Creative Commons Lizenz steht und die betreffende Handlung nicht nach gesetzlichen Vorschriften erlaubt ist, ist für die oben aufgeführten Weiterverwendungen des Materials die Einwilligung des jeweiligen Rechteinhabers einzuholen.

Weitere Details zur Lizenz entnehmen Sie bitte der Lizenzinformation auf http://creativecommons.org/licenses/ by/4.0/deed.de.

\section{Literatur}

Achleitner, S., Huber, A., Lumassegger, S., Kohl, B., Spira, Y., Weingraber, F. (2020): Pilotstudie Oberösterreich - Modellierung von Starkregen-Oberflächenabfluss/Hangwasser - Leitfaden, pp. 1-28. https://rainman-toolbox.eu/wpcontent/uploads/2020/06/AU_Leitfaden.pdf (gesehen 16.November 2021)

Archer, G.E.B., Saltelli, A., Sobol, I.M. (1997): Sensitivity measures, anova-like techniques and the use of bootstrap. I Stat Comput Simul: 58 99-120. https://doi.org/10.1080/009496597088 11825

Bernsteiner, A., Chalupka, A., Eller, M., Englisch, M. Fank, J, Fuchs, G., Gartner, K, Godina, R., Herndl, M., Huber, L., Kammerer, G., Kogelbauer, I., Kohl, B., Kristelly, C., Kuderna, M., Lechner, V., Leitinger, G., Markart, G., Maßwohl, J., Murer, E., Nagl, F., Niedertscheider, K., Nolz, R., Pucher, D., Schulz, K. Stumpp, C. (2020): Wasser im Boden, Sammelband zur quantitativen Erfassung und Auswertung bodenphysikalischer Größen anlässlich 25 Jahre Bodenwasserbeobachtung; Bundesministerium für Landwirtschaft, Regionen und Tourismus, Sektion I - Wasserwirtschaft, Abteilung I/3 - Wasserhaushalt, Marxergasse 2, 1030 Wien. https://info.bmlrt.gv.at/service/ publikationen/wasser/wasser-im-boden-sammelband-zur-quantitativen-erfassungund-auswertung-bodenphysikalischer-gr\% 33 \%B6\%C3\%9Fen-anl\%C3\%A4sslich-25-jahrebodenwasserbeobachtung.html (gesehen 16.November 2021)

Carbone, M., Turco, M., Brunetti, G., Piro, P. (2015): A Cumulative Rainfall Function for Subhourly Design Storm in Mediterranean Urban Areas. Adv Meteorol: 2015, 1-10. https://doi.org/ $10.1155 / 2015 / 528564$

Cheviron, B., Coquet, Y. (2009): Sensitivity Analysis of Transient-MIM HYDRUS-1D: Case Study Related to Pesticide Fate in Soils. Vadose Zone J: 8, 1064. https://doi.org/10.2136/vzj2009.0023

Corradini, C., Melone, F., Smith, R.E. (1994): Modeling infiltration during complex rainfall sequences. Water Resour Res: 30, 2777-2784. https://doi.org/10.1029/94WR00951

Efron, B., Tibshirani, R. (1986): Bootstrap Methods for Standard Errors, Confidence Intervals, and Other Measures of Statistical Accuracy. Stat Sci: 1, 54-75. https://doi.org/10.1214/ss/ 1177013815

van Genuchten, M.T. (1980): A closed-form equation for predicting the hydraulic conductivity of unsaturated soils. Soil Sci Soc Am J: 44, 892-898. https://doi.org/10.2136/sssaj1980. 03615995004400050002x

Glade, T., Mergili, M., Sattler, K. (2020): ExtremA 2019. Aktueller Wissensstand zu Extremereignissen alpiner Naturgefahren in Österreich, Vien- na University Press. https://doi.org/10.14220/ 9783737010924

Goodrich, D.C., Burns, I.S., Unkrich, C.L., Semmens, D.J., Guertin, D.P., Hernandez, M., Yatheendradas, S., Kennedy, J.R., Levick, L.R. (2012): KINEROS2/AGWA: Model Use, Calibration, and Validation. Trans. ASABE: 55, 1561-1574. https://doi.org/10.13031/2013.42264

Houska, T., Multsch, S., Kraft, P., Frede, H.-G., Breuer, L. (2014): Monte Carlo-based calibration and uncertainty analysis of a coupled plant growth and hydrological model. Biogeosciences: 11, 2069-2082. https://doi.org/10.5194/bg-112069-2014

Markart, G., Kohl, B., Sotier, B., Klebinder, K., Schauer, T., Bunza, G., Pirkl, H., Stern, R (2011): A simple code of practice for the assessment of surface runoff coefficients for alpine soil-/vegetation units in torrential rain (version 2.0), Bericht BfW; https://bfw.ac.at/rz/bfwcms. web?dok=10092 (gesehen 16.November 2021); https://doi.org/10.13140/RG.2.1.3406.5441

Meißl, G., Geitner, C., Batliner, A., Klebinder, K., Kohl, B., Markart, G. (2021): Brixenbach research catchment: Quantification of runoff process proportions in a small Alpine catchment depending on soil moisture states and precipitation characteristics. Hydrol Process 35(6): el4186. https://doi.org/10.1002/hyp.14186

Meißl, G., Zieher, T., Geitner, C. (2020): Runoff response to rainfall events considering initial soil moisture-Analysis of 9-year records in a smal Alpine catchment (Brixenbach valley, Tyrol, Austria). J Hydrol Reg Studies 30: 100711. https:// doi.org/10.1016/j.ejrh.2020.100711

Rawls, W.J., Brakensiek, D.L., Saxton, K.E (1982): Estimation of soil water properties. Trans. ASAE 25:1316-1320. https://doi.org/10. $13031 / 2013.33720$

Rezaei, M., Seuntjens, P., Joris, I., Boënne, W., Van Hoey, S., Campling, P., Cornelis, W.M. (2016): Sensitivity of water stress in a twolayered sandy grassland soil to variations in groundwater depth and soil hydraulic parameters. Hydrol Earth Syst Sci: 20, 487-503. https:// doi.org/10.5194/hess-20-487-2016

Ries, F., Kirn, L., Weiler, M. (2020): Runoff reaction from extreme rainfall events on natural hillslopes: a data set from 132 large-scale sprinkling experiments in south-western Germany, Earth Syst Sci Data: 12, 245-255. https://doi.org/10 5194/essd-12-245-2020

Saltelli, A. (2002): Making best use of model evaluations to compute sensitivity indices. Comput Phys Commun: 145, 280-297. https://doi.org/10. 1016/S0010-4655(02)00280-1

Saltelli, A., Annoni, P. (2010): How to avoid a perfunctory sensitivity analysis. Environ Mo- del Softw: 25, 1508-1517. https://doi.org/10 1016/j.envsoft.2010.04.012

Saltelli, A., Annoni, P., Azzini, I., Campolongo, F., Ratto, M., Tarantola, S. (2010): Variance based sensitivity analysis of model output. Design and estimator for the total sensitivity index. Comput Phys Commun: 181, 259-270. https:// doi.org/10.1016/j.cpc.2009.09.018

Saltelli, A., Tarantola, S., Campolongo, F., Ratto, M. (2004): Sensitivity Analysis in Practice: A Guide to Assessing Scientific Models, Wiley, West Sussex.

Šimůnek, J., van Genuchten, M.T., Šejna, M. (2016): Recent Developments and Applications of the HYDRUS Computer Software Packages. Vadose Zone J: 15, 25. https://doi.org/10.2136/ vzj2016.04.0033

Smith, R.E. (1990): Analysis of Infiltration through a Two-Layer Soil Profile. Soil Sci Soc Am J: 54, 1219-1227. https://doi.org/10.2136/ sssaj1990.03615995005400050004x

Smith, R.E., Corradini, C., Melone, F. (1993): Modeling infiltration for multistorm runoff events. Water Resour Res: 29, 133-144. https:// doi.org/10.1029/92WR02093

Sobol, I.M. (2001): Global sensitivity indices for nonlinear mathematical models and thei Monte Carlo estimates. Math Comput Simul: 55, 271-280. https://doi.org/10.1016/S03784754(00)00270-6

Steinbrich, A., Leistert, H., Weiler, M. (2021): RoGeR - ein bodenhydrologisches Modell für die Beantwortung einer Vielzahl hydrologischer Fragen. In Korrespondenz Wasserwirtschaft, 14 Jahrgang, Heft Nr. 2, Feb-2021. https://doi.org/ 10.3243/kwe2021.02.004

Usher, W., Herman, J., Hadka, D., Xantares, F. Bernardoct, M.C. (2015): SALib: New documentation, doc strings and installation requirements. https://doi.org/10.5281/zenodo.31316 Wallner, M. (2021): Evaluation of pedotransferfunctions for estimating soil hydraulic properties and groundwater recharge. Masterarbeit, Universität für Bodenkultur Wien, Institut fü Bodenphysik und landeskulturelle Wasserwirtschaft

Zeitfogel, H., Feigl, M., Schulz, K. (2022): Österreichweite Regionalisierung bodenhydraulischer Eigenschaften. Österreichische Wasserund Abfallwirtschaft 74(3-4). https://doi.org/10. 1007/s00506-022-00842-Z

Hinweis des Verlags Der Verlag bleibt in Hinblick auf geografische Zuordnungen und Gebietsbezeichnungen in veröffentlichten Karten und Institutsadressen neutral. 